Pacific Journal of Mathematics

A CHARACTERIZATION OF GROUPS IN TERMS OF TH 


\title{
A CHARACTERIZATION OF GROUPS IN TERMS OF THE DEGREES OF THEIR CHARACTERS
}

\author{
I. M. IsAacs AND D. S. PASSMAN
}

The purpose of this paper is to study properties of groups which are related to the degrees of their absolutely irreducible characters and in particular to the biggest such degree.

Let $p$ be a fixed prime. We say group $G$ has r.x.e (representation exponent $e$ ) if the degrees of all the absolutely irreducible characters of $G$ divide $p^{e}$. If $G$ has a subinvariant abelian subgroup whose index divides $p^{e}$ then by Ito's Theorem, $G$ has r.x.e. We show conversely that if $G$ has r.x.e then $G$ has a subinvariant abelian subgroup whose index divides $p^{4 e}$. While we do not obtain the best possible value for the exponent in the above bound, we do show that it is essentially a linear function of $e$.

We can obtain information about somewhat larger subgroups. We show that a group $G$ with r.x.e has a subgroup $H$ of index $p^{e}$ with $[H: 3(H)] \leqq p^{3 e(e+2)}$, where $3(H)$ is the center of $H$. The latter bound is by no means best possible. However we show by example that a similar result cannot hold in general for subgroups of index less than $p^{e}$.

We study the case $e=1$ in more detail and completely characterize all such groups. This generalizes a result of Amitsur which discusses the $p=2$ situation. We prove that $G$ has r.x.1 if and only if (i) $G$ is abelian, (ii) $G$ has a normal abelian subgroup of index $p$ or (iii) $[G: 3(G)]=p^{3}$.

The previous results apply to rather special groups. We consider the more general case now. We say group $G$ has r.b.n (representation bound $n$ ) if the degrees of all the absolutely irreducible characters of $G$ are $\leqq n$. If $G$ has an abelian subgroup $A$ with $[G: A] \leqq n$ then as is easily seen $G$ has r.b.n. Conversely we show here that there is a finite valued function $h$ with the property that if $G$ has r.b.n then $G$ has an abelian subgroup $A$ with $[G: A] \leqq h(n)$. This result can be viewed as an analog to Jordan's Theorem for complex linear groups of degree $n$.

The analogy between groups with r.b.n and linear groups of degree $n$ can be carried further. We show that if $G$ has r.b.n and if $p$ is a prime with $p>n$ then $G$ has a normal abelian Sylow $p$-subgroup.

Finally we discuss some extensions of the above results to infinite discrete groups.

1. Preliminary remarks. The main tool of this paper is the character theory of finite groups. We record here our notation and

Received March 17, 1964. 
nomenclature.

Let $\chi$ be an irreducible character of a group $G$ and let $\varphi$ be an irreducible character of a subgroup $H$. $\varphi$ induces a character $\varphi^{*}$ of $G$ and $\chi$ restricts down to a character $\chi \mid H$ of $H$. The Frobenius Reciprocity Theorem ([5] Theorem 38.8) then states

$$
\left[\chi, \varphi^{*}\right]_{\theta}=[\chi \mid H, \varphi]_{H}
$$

where [ ] denotes the inner product over the appropriate group.

Suppose $H \Delta G$ ( $H$ is normal in $G)$. Then $G$ acts on the irreducible characters of $H$ by conjugation. That is for $g \in G$ and $x \in H, \varphi^{g}(x)=$ $\varphi\left(g x g^{-1}\right)$. The subgroup $T$ fixing a given irreducible character $\varphi$ is called the inertia group of $\varphi$. Clearly $T \supseteqq H$. If $t=[G: T]$ (the index of $T$ in $G$ ) then $\varphi$ has precisely $t$ distinct conjugates

$$
\varphi=\varphi_{1}, \varphi_{2}, \cdots, \varphi_{t} .
$$

If $\chi$ is a constituent of $\varphi^{*}$ with multiplicity $a$ then ([5] Theorem 49.7)

$$
\chi \mid H=a\left(\varphi_{1}+\varphi_{2}+\cdots+\varphi_{t}\right) .
$$

Let $H \Delta G$. Each character of $G / H$ can be viewed in a natural way as a character of $G$ with kernel containing $H$. Conversely every such character of $G$ comes from a unique one of $G / H$ in this manner. In general we will use the same symbol to denote the character whether viewed in $G$ or $G / H$. The precise situation will always be clear from context.

Finally we denote by $C[G]$ the group algebra of $G$ over the complex numbers $\boldsymbol{C}$.

We mention here a few results which yield information about the degrees of the irreducible characters of $G$ from a knowledge of the structure of the group.

Proposition 1.1. (Ito). Let $A$ be an abelian subinvariant subgroup of $G$. Then the degrees of the irreducible characters of $G$ all divide $[G: A]$.

Usually this is stated with $A$ normal. However the more general statement is true as can be seen from Theorem 53.17 of [5].

Lemma 1.2. Let $H$ be a normal subgroup of $G$ and $\varphi$ an irreducible character of $H$ with conjugates $\varphi=\varphi_{1}, \varphi_{2}, \cdots, \varphi_{t}$. Let $\chi_{1}, \chi_{2}, \cdots, \chi_{r}$ be the distinct irreducible constituents of $\varphi^{*}$. Then if $\chi_{i} \mid H=a_{i}\left(\varphi_{1}+\varphi_{2}+\cdots+\varphi_{t}\right)$ we have $[G: H]=a_{1}^{2} t+a_{2}^{2} t+\cdots+a_{r}^{2} t$. In particular for each $i, a_{i}^{2} t \leqq[G: H]$. 
Proof. We have deg $\chi_{i}=a_{i} t \operatorname{deg} \varphi$. By Frobenius Reciprocity, $\chi_{i}$ occurs in $\varphi^{*}$ with multiplicity $a_{i}$. Hence

$$
[G: H] \operatorname{deg} \varphi=\operatorname{deg} \varphi^{*}=\Sigma a_{i} \operatorname{deg} \chi_{i}=\left(\Sigma a_{i}^{2} t\right) \operatorname{deg} \varphi
$$

and the result follows.

Proposition 1.3. Let $A$ be an abelian subgroup of $G$. Then the degrees of the irreducible characters of $G$ are $\leqq[G: A]$.

Proof. Let $\chi$ be any character of $G$ and let $\lambda$ be a constituent of $\chi \mid A$. Then $\chi$ is a constituent of $\lambda^{*}$ and hence $\operatorname{deg} \chi \leqq \operatorname{deg} \lambda^{*}=$ $[G: A]$.

This result is true more generally without the assumption that $G$ is finite (see Corollary 1 of [1]).

Proposition 1.4. Let $Z=3(G)$ be the center of $G$. Then the degrees of the irreducible characters of $G$ are $\leqq[G: Z]^{1 / 2}$.

Proof. Let $\chi$ be any character of $G$ and $\chi \mid Z=\alpha \Sigma_{1}^{t} \lambda_{i}$. Since $Z$ is the center of $G$ we must have $t=1$. Thus

$$
\operatorname{deg} \chi=a=\left(a^{2} t\right)^{1 / 2} \leqq[G: Z]^{1 / 2}
$$

and the result follows.

This paper is concerned with the converse problem, namely to obtain information about the structure of the group from the degrees of its characters. We use the above propositions as guide lines to indicate the directions in which to look.

2. Extra-special groups. We study here the structure and characters of groups $E$ with the following properties.

Definition 2.1. We say a group $E$ is extra-special if

(i) $E$ is a non-abelian group containing a nontrivial normal abelian subgroup.

(ii) All nontrivial normal subgroups of $E$ contain the commutator subgroup $E^{\prime}$.

We mention first how these extra-special groups occur. Let $G$ be any non-abelian group. Let $K$ be normal in $G$ and maximal with respect to $G / K$ being non-abelian. Then clearly $E=G / K$ satisfies (ii). If for some reason (for example the solvability of $G$ ) we can guarantee that $E$ has a nontrivial normal abelian subgroup, then $E$ is extra- 
special.

Proposition 2.2. Let $E$ be an extra-special group. Then $E$ has the properties of one of the following two cases:

Case $P:$ (i) $E$ is a $p$-group for some prime $p$.

(ii) $3(E)$ is cyclic and $E^{\prime}$ has order $p$ and is contained in $Z=$ 3(E).

(iii) if $\beta$ is any nonlinear absolutely irreducible character of $E$ then $\beta$ vanishes off $Z$ and $\operatorname{deg} \beta=[E: Z]^{1 / 2}$.

Case $Q$ : (iv) $E$ is not a $p$-group for any prime $p$.

(v) $E^{\prime}=Q$ is a normal, elementary abelian Sylow $q$-subgroup of $E$ with $E / Q$ cyclic.

(vi) every nonlinear character of $E$ has degree $[E: Q]$.

(vii) if $\lambda$ is a nonprincipal character of $Q$ then $\lambda$ has $[E: Q]$ distinct conjugates in $E$. For any linear character $\lambda$ of $Q$ and any element $g \in E-Q$ there exists a character $\mu$ of $Q$ with $\lambda=\mu^{g} \bar{\mu}$.

Proof. First suppose $E$ is a $p$-group so we have (i) of Case $P$. Let $Y$ be a cyclic subgroup of order $p$ contained in the center $Z=$ $3(E)$. Since $Y$ is normal in $E$ we have $Y \supseteqq E^{\prime}$. But $E^{\prime}>1$ so we in fact have $Y=E^{\prime}$ and $\left|E^{\prime}\right|=p$. Thus $Z$ has one and only one such cyclic subgroup of order $p$ and so $Z$ is cyclic. This yields (ii).

Now let $\beta$ be a nonlinear character of $E$ and let $g \notin Z$. Since $g$ is not central there is an element $h$ with $(g, h)=g^{-1} h^{-1} g h \neq 1$. But $(g, h) \in E^{\prime} \subseteq Z$ so say $(g, h)=z$. Then $h^{-1} g h=g z$. Since $\beta$ is a class function we have $\beta(g)=\beta\left(h^{-1} g h\right)=\beta(g z)$. Now $z \in Z$ so $z$ is represented by the scalar matrix $\lambda I$ in the representation associated with $\beta$. If $\lambda=1$ then $z$ and hence $E^{\prime}$ is in the kernel of the representation. Since this is not the case $\lambda \neq 1$. But clearly $\beta(g z)=\lambda \beta(g)$ so we have $\beta(g)=\lambda \beta(g)$ and hence $\beta(g)=0$. Finally for any $z \in Z$ we have clearly $|\beta(z)|=b$ where $b=\operatorname{deg} \beta$. Hence

$$
1=[\beta, \beta]=|G|^{-1} \Sigma_{G} \beta(g) \overline{\beta(g)}=|G|^{-1} \Sigma_{Z} \beta(g) \overline{\beta(\bar{g})}=b^{2}|Z| /|G| .
$$

Thus $b^{2}=[G: Z]$ and (iii) follows.

Now we assume that $E$ is not a $p$-group for any prime $p$. Let $A$ be the nontrivial normal abelian subgroup of $E$ guaranteed by Definition 2.1. If $|A|$ has two distinct prime factors say $p$ and $q$ then $\mathfrak{S}_{p}(A)$ (the Sylow $p$-subgroup of $A$ ) and $\mathfrak{S}_{q}(A)$ are characteristic in $A$ and hence normal in $E$. Thus $\mathfrak{S}_{p}(A) \supseteqq E^{\prime}$ and $\mathfrak{S}_{q}(A) \supseteqq E^{\prime}$ so $E^{\prime}=1$, a contradiction. Hence $A$ is a $q$-group for some prime $q$. Let $Q$ be a Sylow $q$-subgroup of $E$ containing $A$. Since $A \supseteqq E^{\prime}$ we see that $Q$ is normal in $E$. Now $E$ is solvable so by a theorem of P. Hall ([7] Theorem 9.3.1) $Q$ has a complement $B$ which is abelian since $B \simeq E / Q$. 
We show first that $Q$ is elementary abelian. Let $\Phi(Q)$ be the Frattini subgroup of $Q$. Suppose that $\Phi(Q)>1$. Then since $\Phi$ is characteristic in $Q$ it is normal in $E$ and hence $\Phi(Q) \supseteqq E^{\prime}$. Thus $(B, Q) \subseteq \Phi(Q)$ so $B$ has trivial action on $Q / \Phi(Q)$, the Frattini quotient of $Q$. But $B$ is a $q^{\prime}$-group so ([7] Theorem 12.2.2) $B$ centralizes $Q$ and $E=B \times Q$. Hence since $B>1, E^{\prime} \subseteq B \cap Q=1$, a contradiction. Thus $\Phi(Q)=1$ and $Q$ is elementary abelian.

Now $\mathfrak{F}(Q) \supseteqq Q$ where $\mathfrak{C}(Q)$ is the centralizer of $Q$. Suppose $\mathfrak{G}(Q)>Q$. Then if $C$ is a complement for $Q$ in $\mathfrak{S}(Q)$ we have $\mathbb{S}(Q)=$ $Q \times C$ and so $C=\mathfrak{S}_{q^{\prime}}(\mathfrak{C}(Q))$. Thus $C$ is normal in $E$ and again $E^{\prime} \subseteq Q \cap C=1$, a contradiction. Thus $Q$ is its own centralizer. Let $g \notin Q$ and set $D=\langle Q, g\rangle$. Now $3(D) \subseteq Q$ and this is clearly the set of fixed points in $Q$ under the action of $g$. Note since $Q$ is abelian we have a well-defined action of $E / Q$ on $Q$. Since $D \supset Q \supseteqq E^{\prime}, D$ and hence $3(D)$ is normal in $E$. If $3(D)>1$ then $3(D) \supseteqq E^{\prime} \supseteqq D^{\prime}$ and $D$ is nilpotent. But this would imply that $g$ centralizes $Q$, a contradiction. Thus $3(D)=1$ and so $g$ acts fixed point free on $Q$. Hence $E / Q$ acts fixed point free on $Q$ and $E$ is a Frobenius group with kernel $Q$. Since $E / Q$ is abelian, it is cyclic ([4] Theorem $\mathrm{V}$, page 336). Let $x, y \in Q$ and $g \notin Q$. Then $x^{o} x^{-1}=y^{o} y^{-1}$ implies $\left(x y^{-1}\right)^{o}=x y^{-1}$ so that $x=y$. Hence the $|Q|$ elements $x^{g} x^{-1}$ for all $x \in Q$ are all distinct. Thus $Q=E^{\prime}$ and (v) follows.

Let $\lambda$ be a linear character of $Q$. Suppose for some $g \notin Q$ we have $\lambda^{g}=\lambda$. Then for all $x \in Q, \lambda(x)=\lambda\left(g x g^{-1}\right)$ so that $\lambda\left(g x g^{-1} x^{-1}\right)=$ 1. But as above $g x g^{-1} x^{-1}$ takes on all elements of $Q$ as $x$ does. So $\lambda$ is in fact the principal character of $Q$. Hence any non-principal character has $[E: Q]$ distinct conjugates. Since the characters $\mu^{\theta} \bar{\mu}$ are all distinct (vii) follows. Finally let $\beta$ be a nonlinear character of $E$. Since $Q=E^{\prime}, \beta \mid Q$ has a nonprincipal character as a constituent. But this has $[E: Q]=t$ distinct conjugates. Hence $\beta \mid Q=a \Sigma_{1}^{t} \lambda_{i}$. Since $a^{2} t \leqq[E: Q], a=1$ and $\operatorname{deg} \beta=a t=[E: Q]$. This completes the proof.

We remark that in the proof of (iii) above we actually obtained the more general result:

Lemma 2.3. Let $F$ be a class 2 group with $\left|F^{\prime}\right|=p$. If $\beta$ is an irreducible nonlinear character of $F$ then $\operatorname{deg} \beta=[F: 3(F)]^{1 / 2}$.

\section{Groups with r.x.e.}

Definition 3.1. Let $p$ be a fixed prime. We say a group $G$ has r.x.e (representation exponent $e$ ) if the degrees of all absolutely irreducible characters of $G$ divide $p^{e}$. We say a group $G$ has r.x. $(e, s)$ 
if $G$ has r.x.e and $\mathfrak{S}_{p}(G)$ has r.x.s.

Let $f$ be the function with the following property. If $G$ has r.x.e then $G$ has a subinvariant abelian subgroup whose index in $G$ divides $p^{f(e)}$. Moreover $f$ is the smallest such function.

Let $g$ be the function with the following property. If $G$ has r.x. $(e, s)$ then $G$ has a subinvariant abelian subgroup whose index in $G$ divides $p^{g(e, s)}$. Moreover $g$ is the smallest such function.

There is of course no apriori reason to assume that finite valued functions $f$ and $g$ exist. We show below that they do exist and give bounds for their values.

THEOREM A 3.2. The functions $f$ and $g$ exist and satisfy

$$
\begin{array}{ll}
f(0)=0 & 2 e \leqq f(e) \leqq 4 e-\log _{2} 4 e \\
g(e, 0)=e & e+\min (e, s) \leqq g(e, s) \leqq e+3 s-\log _{2} 4 s .
\end{array}
$$

This yields in particular

$$
\begin{aligned}
& f(0)=0, \quad f(1)=2, \quad f(2)=4 \text { or } 5 \\
& g(e, 0)=e, \quad g(e, 1)=e+1, \quad e+2 \leqq g(e, 2) \leqq e+3 .
\end{aligned}
$$

We will show in a later paper that $f(2)=4$.

Let $\varphi$ be an irreducible character of $\mathfrak{S}_{p}(G)$ and $\chi$ a constituent of $\varphi^{*}$. Then $\varphi$ is a constituent of $\chi \mid \mathscr{S}_{p}(G)$ so we have $\operatorname{deg} \varphi \leqq \operatorname{deg} \chi$. If $G$ has r.x.e then $\operatorname{deg} \chi \leqq p^{e}$. But $\Im_{p}(G)$ is a $p$-group so the degrees of all its characters are powers of $p$ and so $\mathfrak{S}_{p}(G)$ has r.x.e. Thus $G$ has r.x. $(e, e)$ and it clearly suffices to consider only the function $g$.

LEMma 3.3. Let $N \Delta G$. If $G$ has r.x. $(e, s)$ then so does $N$ and $G / N$.

Proof. Let $\varphi$ be an irreducible character of $N$ and $\chi$ a constituent of $\varphi^{*}$. Then since $N$ is normal $\chi \mid N=a \Sigma_{1}^{t} \varphi_{i}$ where the $\varphi_{i}$ are the $t$ distinct conjugates of $\varphi$. Hence $\operatorname{deg} \chi=a t \operatorname{deg} \varphi$. Since $\operatorname{deg} \chi$ divides $p^{e}$ so does $\operatorname{deg} \varphi$ and $N$ has r.x.e.

Choose a Sylow $p$-subgroup of $N$ and extend it to one of $G$. Thus $\mathfrak{S}_{p}(N) \cong \mathfrak{S}_{p}(G)$. Let $\varphi$ be a character of $\mathfrak{S}_{p}(N)$ and $\chi$ a constituent of $\varphi^{*}$ (induction to $\mathfrak{S}_{p}(G)$ ). Then $\varphi$ is a constituent of $\chi \mid \Im_{p}(N)$ and so $\operatorname{deg} \varphi \leqq \operatorname{deg} \chi$. Since $\operatorname{deg} \varphi$ is a power of $p$ and $\operatorname{deg} \chi \leqq p^{s}$, the first result follows. The second is obvious.

Proposition 3.4. Let $G$ have r.x. $(e, s)$. Then $G$ has a normal abelian Hall $p^{\prime}$-subgroup $\mathfrak{S}(G)$.

Proof. We show first by induction on $|G|$ that $G$ has a normal 
$\mathfrak{g}(G)$. If $G$ is a $p^{\prime}$-group the result is of course trivial. So we assume that $p$ divides $|G|$. From the equation

$$
|G|=\left[G: G^{\prime}\right]+\Sigma_{i} x_{i}^{2}
$$

where the $x_{\imath}^{2}$ are the degrees of the nonlinear irreducible characters of $G$ we can conclude that $p$ divides $\left[G: G^{\prime}\right]$. Let $K$ be the complete inverse image in $G$ of a subgroup of index $p$ in the abelian group $G / G^{\prime}$. Then $K \Delta G$ so by the previous lemma $K$ has r.x. $(e, s)$. By

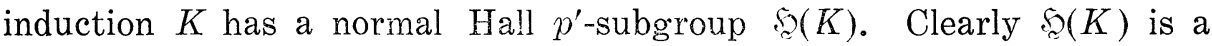
Hall $p^{\prime}$-subgroup of $G$ and since it is characteristic in $K$, it is normal in $G$. Hence $G$ has a normal $g(G)$.

Since $S_{(}(G)$ has r.X. $_{0}(e, s)$ all its characters have degrees which are powers of $p$. But $|\mathfrak{g}(G)|$ is prime to $p$ so all the characters are linear and $\mathfrak{g}(G)$ is abelian. This completes the proof.

Lemma 3.5. Let $G$ be an arbitrary group. Suppose $K \Delta G$ with $E=G / K$ being an extra-special case $P$ group. Let $Z$ be the complete inverse image in $G$ of the center of $E$. Let $\beta$ be a nonlinear character of $E$ viewed in $G$. Given any absolutely irreducible character $\varphi$ of $Z$, if $\chi$ is a constituent of $\varphi^{*}$ and if $\chi_{1}$ is a constituent of $\chi \beta$ then

$$
(\operatorname{deg} \chi)\left(\operatorname{deg} \chi_{1}\right) \geqq p^{m} t(\operatorname{deg} \varphi)^{2}
$$

where $t$ is the number of distinct conjugates of $\varphi$ and $\operatorname{deg} \beta=p^{m}$.

Proof. If $\operatorname{deg} \beta=p^{m}$ then by (iii) of Proposition 2.2 we have $[G: Z]=p^{2 m}$. Also $\beta \mid Z=p^{m} \lambda$ where $\lambda$ is a linear character of $Z / K$. Moreover since $Z / K$ is central in $G / K, \lambda$ is invariant under the action of $G$. That is $\lambda^{x}=\lambda$ for all $x \in G$.

Let $\chi$ be a constituent of $\varphi^{*}$. Then since $Z \Delta G$

$$
\chi \mid Z=a \Sigma_{1}^{t} \varphi_{i} \quad \varphi_{1}=\varphi .
$$

Now $\chi \beta$ vanishes off $Z$ by (iii) again and

$$
\chi \beta \mid Z=a p^{m} \Sigma_{1}^{t} \varphi_{i} \lambda \text {. }
$$

Also $(\varphi \lambda)^{*}$ vanishes off $Z$ and

$$
(\varphi \lambda)^{*} \mid Z=\left(p^{2 m} / t\right) \Sigma_{1}^{t} \varphi_{i} \lambda \text {. }
$$

The first is clear. The second follows since $(\varphi \lambda)^{x}=\varphi^{x} \lambda^{x}=\varphi^{x} \lambda$ and $\operatorname{deg}(\varphi \lambda)^{*}=[G: Z] \operatorname{deg} \varphi \lambda=p^{2 m} \operatorname{deg} \varphi \lambda$. Thus we have

$$
\chi \beta=a t p^{-m}(\varphi \lambda)^{*} \text {. }
$$

Say $(\varphi \lambda)^{*}=\Sigma a_{i} \chi_{i}$. Then $\chi \beta=\Sigma a_{i} a t p^{-m} \chi_{i}$ and hence $a_{i} a t \geqq p^{m}$. In 
particular $a_{1} a t \geqq p^{m}$. By reciprocity $\chi_{1} \mid Z=a_{1} \Sigma_{1}^{t} \varphi_{i} \lambda$ so we have $\operatorname{deg} \chi=a t \operatorname{deg} \varphi, \operatorname{deg} \chi_{1}=a_{1} t \operatorname{deg} \varphi \lambda=a_{1} t \operatorname{deg} \varphi$.

Hence

$$
(\operatorname{deg} \chi)\left(\operatorname{deg} \chi_{1}\right)=a a_{1} t^{2}(\operatorname{deg} \varphi)^{2} \geqq p^{m} t(\operatorname{deg} \varphi)^{2}
$$

and the result follows.

We are now ready to prove the theorem.

Proof of Theorem A. The main difficulty is the upper bound. We show this by induction on $|G|$.

Since $G / \mathfrak{g}(G) \simeq \mathfrak{S}_{p}(G)$ the second parameter in r.x. $(e, s)$ can be viewed as a bound for the characters of $G / \mathfrak{S}(G)$ and this is the approach we take. These of course correspond to the characters of $G$ containing $\mathfrak{S}(G)$ in their kernel. Since the upper bound for the function $g$ is always nonnegative we see that the result holds if $G$ is abelian.

So we assume $G$ is nonabelian. Choose $K \Delta G$ maximal with respect to $G / K$ being nonabelian. Such a subgroup of course exists. Now $E=G / K$ always has a normal abelian subgroup by Proposition 3.4 , hence $E$ is extra-special. By Propositions 3.4 and 2.2 we see that $E$ is either a $p$-group or a Frobenius group where $E / E^{\prime}$ is a cyclic $p$ group. This yields two cases to study. We discuss the latter first.

Case $Q$. Let $Q$ be the complete inverse image in $G$ of $E^{\prime}$, the Sylow $q$-subgroup of $E$. Then $[G: Q]=\left[E: E^{\prime}\right]=p^{m}$ for some integer $m$. Let $\lambda$ be a fixed linear character of $Q$ which corresponds to a nonprincipal linear character of $E^{\prime}=Q / K$.

Now let $\theta$ be any character of $Q$ and let $T(\theta)$ be its inertia group. The product $\theta \lambda$ is also an absolutely irreducible character of $Q$. Suppose both $T(\theta)>Q$ and $T(\theta \lambda)>Q$. Since $G / Q \simeq E / E^{\prime}$ is a cyclic $p$ group there is a unique minimal subgroup of $G$ containing $Q$. Hence we can find $x \notin Q$ with $x \in T(\theta) \cap T(\theta \lambda)$. Then

$$
\theta \lambda=(\theta \lambda)^{x}=\theta^{x} \lambda^{x}=\theta \lambda^{x}
$$

so $\theta=\theta \lambda^{x} \bar{\lambda}$. But $x \notin Q$ so $\mu=\lambda^{x} \bar{\lambda}$ is not the principal character of $E^{\prime}$. If $N$ denotes its kernel in $Q$ then $Q>N \supseteqq K, N \Delta Q$ and since $\theta=\theta \mu, \theta$ vanishes off $N$. Say $\theta \mid N=a \Sigma_{1}^{t} \varphi_{i}$ with $\varphi_{1}=\varphi$. Then deg $\theta=a t \operatorname{deg} \varphi$. Since $\theta$ vanishes of $N$

$$
1=[\theta, \theta]_{Q}=(|N| /|Q|)[\theta|N, \theta| N]_{N}=(|N| /|Q|) a^{2} t
$$

so $a^{2} t=[Q: N]$. Now $a$ and $t$ divide $\operatorname{deg} \theta$, a power of $p$. But they also divide $[Q: N]$ which is prime to $p$. Hence $a=t=1$ and $[Q: N]=$ 
1 , a contradiction.

Thus either $T(\theta)=Q$ or $T(\theta \lambda)=Q$ or both. Since $\operatorname{deg} \theta \lambda=\operatorname{deg} \theta$, it sufficies to assume $T(\theta)=Q$. Let $\chi$ be a constituent of $\theta^{*}$. Then $\chi \mid Q=b \Sigma \theta_{i}$ where the $\theta_{i}$ are the $p^{m}$ distinct conjugates of $\theta$. So $p^{m} \operatorname{deg} \theta$ divides $\operatorname{deg} \chi$. Since $G$ has r.x.e, $Q$ therefore has r.x. $(e-m)$. But $Q$ also has r.x. $(e, s)$ by Lemma 3.3. Hence $Q$ has r.x. $(e-m, s)$. Since $|Q|<|G|$ we have by induction a subinvariant abelian subgroup $A$ of $Q$ of index $p^{n}$ with $n \leqq e-m+3 s-\log _{2} 4 s$ (or $n \leqq e-m$ if $s=$ $0)$. Then $A$ is subinvariant in $G$ with index $p^{m+n}$ and here

$$
m+n \leqq e+3 s-\log _{2} 4 s \quad(\text { or } m+n \leqq e) \text {. }
$$

Thus induction goes through in this case.

Case $P$. Let $Z$ be the complete inverse image in $G$ of the center of $E$. Let $\beta$ be a nonlinear character of $E$ viewed in $G$ so that deg $\beta=p^{m}$. If $\varphi$ is a character of $Z$ then by Lemma 3.5

$$
(\operatorname{deg} \varphi)^{2} \leqq 1 / p^{m} t(\operatorname{deg} \chi)\left(\operatorname{deg} \chi_{1}\right) \leqq p^{2 e-m} / t
$$

If in fact $\varphi$ is a character of $Z / \mathfrak{S}(Z)$ then since $\mathscr{S}(Z)=\mathfrak{S}(G)$ and $\beta$ is a character of $G / S_{2}(G)$ we see that so are both $\chi$ and $\chi_{1}$. Hence

$$
(\operatorname{deg} \varphi)^{2} \leqq 1 / p^{m} t(\operatorname{deg} \chi)\left(\operatorname{deg} \chi_{1}\right) \leqq p^{2 s-m} / t
$$

Thus clearly $Z$ has r.x. $(e-m / 2, s-m / 2)$.

If $m$ is odd we do slightly better. If $\operatorname{deg} \varphi=p^{r}$ then $r \leqq e-m / 2$ implies $r \leqq e-(m+1) / 2$. Hence we have:

(*) If $m$ is odd $G$ has a normal subgroup $Z$ with $[G: Z]=p^{2 m}$ and such that $Z$ has r.x. $(e-(m+1) / 2, s-(m+1) / 2)$.

Now let $m$ be even. Let $B>Z$ with $[B: Z]=p$. Since $B / K$ is normal in $G / K, B$ is normal in $G$. We show that $B$ has r.x. $(e-m / 2$, $s-m / 2)$. Both the $e$ and the $s$ terms follow in the same manner so it suffices to just discuss the $e$ term here. Suppose $B$ has a character $\theta$ of degree $>p^{e-m / 2}$. Let $\theta \mid Z=a \Sigma_{1}^{r} \varphi_{i}$. Since $a^{2} r \leqq[B: Z]=p$ by Lemma 1.2 and both $a$ and $r$ are powers of $p$ it follows that $a=1$ and $r=1$ or $p$. But $\operatorname{deg} \varphi_{1} \leqq p^{e-m / 2}$ by the above so we must have $r=p$ and $\operatorname{deg} \varphi_{1}=p^{e-m / 2}$. Now $\varphi_{1}$ has at least $p$ conjugates in $B$. Hence if $\varphi_{1}$ has $t$ conjugates in $G$ we have $t \geqq r=p$. But finally

$$
p^{2 e-m}=\left(\operatorname{deg} \varphi_{1}\right)^{2} \leqq p^{2 e-m} / t \leqq p^{2 e-m-1},
$$

a contradiction. Thus we have:

$(* *)$ If $m$ is even $G$ has a normal subgroup $B$ with $[G: B]=p^{2 m-1}$ and such that $B$ has r.x. $(e-m / 2, s-m / 2)$. 
Suppose $m$ is odd. By induction and $\left(^{*}\right) Z$ has a subinvariant abelian subgroup $A$ with $[Z: A]=p^{n}$ where

$$
n \leqq(e-(m+1) / 2)+3(s-(m+1) / 2)-\log _{2} 4(s-(m+1) / 2) .
$$

But $A$ is subinvariant in $G$ with $[G: A]=p^{n+2 m}$ and

$$
\begin{aligned}
& n+2 m \leqq e+3 s-2-\log _{2} 4(s-(m+1) / 2) \\
& \quad=e+3 s-\log _{2} 4(4 s-2 m-2) .
\end{aligned}
$$

Clearly $s \geqq m$ since $\operatorname{deg} \beta=p^{m}$ so $4 s-2 m-2 \geqq 2 s-2 \geqq s$ for $s \geqq 2$ and the result follows here. If $s=m=1$ then $n \leqq e-1$ so

$$
n+2 m \leqq e+1=e+3 s-\log _{2} 4 s
$$

so the result follows.

Finally let $m$ be even. By induction and $\left({ }^{*}\right) B$ has a subinvariant abelian subgroup $A$ with $[B: A]=p^{n}$ where

$$
n \leqq(e-m / 2)+3(s-m / 2)-\log _{2} 4(s-m / 2) .
$$

But $A$ is subinvariant in $G$ with $[G: A]=p^{2 m-1+n}$ and

$$
\begin{aligned}
2 m-1+n & \leqq e+3 s-1-\log _{2} 4(s-m / 2) \\
& =e+3 s-\log _{2} 4(2 s-m) \\
& \leqq e+3 s-\log _{2} 4 s
\end{aligned}
$$

since $s \geqq m$. Thus the result follows and the upper bound in Theorem $A$ is proved.

For the lower bound we need the following.

$$
\begin{array}{ll}
\text { LEMmA 3.6. } & \text { (i) } f(1) \geqq 2 \\
& \text { (ii) } f\left(e_{1}+e_{2}\right) \geqq f\left(e_{1}\right)+f\left(e_{2}\right) \\
& \text { (iii) } g(e, s) \geqq e-s+f(s) \quad \text { for } e \geqq s .
\end{array}
$$

Proof. Let $G_{i}$ be a group with r.x. $_{0} e_{i}$ which has no subinvariant abelian subgroup with index $p^{f\left(e_{i}\right)-1}$. Then $G_{1} \times G_{2}$ has r.x. $\left(e_{1}+e_{2}\right)$ and has no subinvariant abelian subgroup whose index divides $p^{f\left(e_{1}\right)+f\left(e_{2}\right)-1}$. Hence $f\left(e_{1}+e_{2}\right) \geqq f\left(e_{1}\right)+f\left(e_{2}\right)$ and (ii) is proved.

Let $G$ be a group with r.x.s which has no subinvariant abelian subgroup with index dividing $p^{f(s)-1}$. Let $Q$ be an elementary abelian $q$-group with $p^{e-s}$ generators and let $H$ be the semi-direct product of $Q$ with an element of order $p^{e-s}$ where the element merely cyclically permutes a basis of $Q$. Clearly $Q$ is a maximal abelian subgroup of $H$. By Proposition 1.1, $H$ has r.x. $(e-s, 0)$ since $\mathfrak{S}_{p}(H)$ is cyclic. Then $G \times H$ has r.x. $(e, s)$ but has no subinvariant abelian subgroup whose index divides $p^{e-s+f(s)-1}$. Thus $g(e, s) \geqq e-s+f(s)$ and (iii) follows. 
Part (i) is a consequence of Example 3.7 below.

EXAMPLE 3.7. Let $F_{1}$ be the group of order $p^{6}$ generated by elements $u, v, w, x, y, z$ all of period $p$ with the additional relations: $x, y$, and $z$ are central and

$$
(u, v)=x, \quad(v, w)=y, \quad(w, u)=z .
$$

It is not hard to see that $F_{1}$ has no abelian subgroup of index $p$ and that $\left[F_{1}: 3\left(F_{1}\right)\right]=p^{3}$. By Proposition 1.4, $F_{1}$ has r.x.1.

We can now finish the proof of Theorem A. Since $f(1) \geqq 2$ we have by (ii) of Lemma 3.6 and induction that $f(e) \geqq 2 e$. Then by (iii) of Lemma 3.6 if $e \geqq s$ we have $g(e, s) \geqq e$-s $+f(s) \geqq e-s+2 s=e+s$. If $G$ has r.x. $(e, s)$ and $s>e$ then clearly $G$ has r.x. $(e, e)$ so that $g(e, s)=g(e, e)$ in this case and the result follows.

While we do not have the exact value for the function $f$, the following may be of interest.

Proposition 3.8. There exists a real number $c$ with $2 \leqq c \leqq 4$ and $f(e) \sim c e$.

Proof. Set $\mu(e)=f(e) / e-f(1) / 1$. By Theorem A, $2 \geqq \mu(e) \geqq 0$. Clearly it suffices to show that $\mu(e)$ has a limit as $e \rightarrow \infty$. By Lemma 3.6, $f\left(e_{1}\right)+f\left(e_{2}\right) \leqq f\left(e_{1}+e_{2}\right)$ so we obtain

$$
\begin{aligned}
& \mu(e+1) \geqq e /(e+1) \mu(e) \\
& \mu(n e) \geqq \mu(e) \text { for all positive integers } n .
\end{aligned}
$$

Fix $e \geqq 1$ and let $x$ be any positive integer. Say $(n+1) e \geqq x>n e$. Then by $(*)$ and $(* *)$.

$$
\begin{aligned}
\mu(x) & \geqq\left(\frac{x-1}{x}\right)\left(\frac{x-2}{x-1}\right) \cdots\left(\frac{n e}{n e+1}\right) \mu(n e) \\
& =\left(\frac{n e}{x}\right) \mu(n e) \geqq \frac{n}{n+1} \mu(e) .
\end{aligned}
$$

As $x \rightarrow \infty, n \rightarrow \infty$ and so $\lim \inf \mu(x) \geqq \mu(e)$. Since this is true for all $e$, $\lim \inf \mu(x) \geqq \lim \sup \mu(x)$ and by the boundedness of $\mu$ the limit exists.

We will need an additional result for our later study of groups with r.x.1. Since the abelian group occurs on the first step in this case in the inductive proof of Theorem A we obtain

Lemma 3.9. Let $G$ have r,x,1. Then $G$ has a normal abelian. 
subgroup whose index divides $p^{2}$.

4. Large centers. Let $G$ be a group with r.x.e. In the previous section we showed that some subgroup of index $p^{n}$ with $2 e \leqq n \leqq 4 e$ is abelian. We now study subgroups of index $p^{e}$.

THEOREM B 4.1. Let $G$ be a group with r.x.e. Then $G$ has a subgroup $H$ of index $p^{e}$ with $[H: 3(H)] \leqq p^{3 e(e+2)}$.

We first need certain counting formulas.

Definition 4.2. Let $A$ be an abelian subgroup of an arbitrary group $G$. Group $A$ acts on $G$ by conjugation and we call the orbits of this action the $A$-classes of $G$. Clearly $G$ is partitioned into mutually disjoint $A$-classes. We denote by $r(A)$ the number of such classes.

If $A$ is normal then $G$ acts by conjugation on the linear characters of $A$. We denote by $s(A)$ the number of orbits in this action.

Lemma 4.3. Let $G$ be a group with r.x.e. and let $A$ be an abelian subgroup. Let $k_{1}, k_{2}, \cdots, k_{r}$ denote the A-classes of $G$ with $r=r(A)$ and let $x_{j}$ be a representative of $k_{j}$. Then

(i) $\left|k_{j}\right|=\left[A: \varsigma_{A}\left(x_{j}\right)\right]$

(ii) $\left|k_{1}\right|+\left|k_{2}\right|+\cdots+\left|k_{r}\right|=|G|$

(iii) $r(A) \geqq|G| / p^{e}$ with strict inequality if $G$ is nonabelian. If $A$ is normal we have in addition

(iv) $r(A)=[G: A] s(A)$.

Proof. Formulas (i) and (ii) are clear. Let $C[G]$ be the group algebra of $G$ over the complex numbers and let $A$ be naturally embedded. We consider $\mathscr{C}(A)$, the centralizer of $A$ in the group algebra. Let $\hat{k}_{j}$ denote the sum of the elements of $k_{j}$ in $C[G]$. It is clear that $\hat{k}_{1}, \hat{k}_{2}, \cdots, \hat{k}_{r}$ form a basis for $\mathscr{C}(A)$ so that $r(A)=\operatorname{dim} \mathscr{C}(A)$.

We now compute this dimension in another manner. $C(G)$ is a direct sum of full matrix rings over the complex numbers. Say

$$
C[G]=\cdot \sum_{i=0}^{e} b_{i}[C]_{p i}
$$

since $G$ has r.x.e. In any such direct summand $[C]_{p^{i}}$ the projection of $A$ can be diagonalized. Thus $\mathscr{C}(A)$ contains all diagonal matrices in each term. Hence

$$
\operatorname{dim} \mathscr{C}(A) \geqq \sum b_{i} p^{i}
$$




$$
|G|=\sum b_{i} p^{2 i} \leqq p^{e} \sum b_{i} p^{i}
$$

with strict inequality if $G$ is non-abelian since then $e \geqq 1$ and $b_{0} \geqq 1$. Thus $r(A)=\operatorname{dim} \mathscr{C}(A) \geqq|G| / p^{e}$ and (iii) follows.

If $A$ is normal we can compute the dimension more precisely. We consider one of the direct summands of the group algebra and assume $A$ is diagonalized in it. Let $\chi$ be the character of this representation with $\chi \mid A=a \Sigma_{1}^{t} \lambda_{i}$. We write $a=a(\chi)$ and $t=t(\chi)$ to indicate their dependence on $\chi$. It is easy to see that the dimension of the centralizer of $A$ is equal to $a^{2} t$. Hence

$$
\operatorname{dim} \mathscr{C}(A)=\sum_{x} a(\chi)^{2} t(\chi) .
$$

In view of Lemma 1.2 , we obtain by grouping these terms

$$
r(A)=\operatorname{dim} \mathscr{C}(A)=[G: A] s(A)
$$

and the proof is complete.

Lemma 4.4. Let $G$ be a group with r.x.e and let $A$ be an abelian subgroup. Suppose $[G: \mathbb{C}(A)] \geqq p^{e+1}$. Then there exists an element $x \notin \Subset(A)$ with

$$
\left[A: \mathfrak{c}_{A}(x)\right]<\left(p^{e+1}-1\right) /(p-1)=p^{e}+p^{e-1}+\cdots+1<p^{e+1} .
$$

If $G$ is a p-group then $\left[A: \mathfrak{E}_{A}(x)\right] \leqq p^{e}$.

Proof. Let $n=\min \quad\left\{\left[A: \mathfrak{C}_{A}(x)\right] \mid x \notin \subseteq(C A)\right\}$. We show that $n<\left(p^{e+1}-1\right) /(p-1)$.

If $g \in \mathbb{C}(A)$ then the $A$-class containing $g$ has only one element and there are $|\mathfrak{S}(A)|$ such classes. If $g \notin \mathfrak{C}(A)$ then the $A$-class containing $g$ has at least $n$ members by definition of $n$. Hence there are at most $(|G|-|\mathfrak{C}(A)|) / n$ such classes. By the previous lemma we have

$$
|\mathfrak{S}(A)|+(|G|-|\mathfrak{S}(A)|) / n \geqq r(A)>|G| / p^{e}
$$

since clearly $G$ is non-abelian. Let $a=[G: \subseteq(A)]$. Then the above equation becomes

$$
1+(a-1) / n>a / p^{e}
$$

or $n / p^{e}<(a-1) /\left(a-p^{e}\right) \leqq\left(p^{e+1}-1\right) /\left(p^{e+1}-p^{e}\right)$ since $a \geqq p^{e+1}$. Hence $n<\left(p^{e+1}-1\right) /(p-1)$. If $G$ is a $p$-group then $n$ must be a power of $p$ and so we have $n \leqq p^{e}$ in this case. This completes the proof.

Proof of Theorem $B$. We show by induction on $i$ with $0 \leqq i \leqq 3 e$ that $G$ has a subgroup $H_{i}$ of index $p^{4 e-i}$ with $\left[H_{i}: 3\left(H_{i}\right)\right] \leqq p^{i(e+2)}$. By 
Theorem A we have the group $H_{0}$.

Suppose we have $H_{i}$ with $i<3 e$. Let $A=3\left(H_{i}\right)$. Suppose first that $[G: \subseteq(A)] \leqq p^{e}$. Then we can choose $H_{i+1}$ with $\subseteq(A) \supseteqq H_{i+1}>H_{i}$ with $\left[H_{i+1}: H_{i}\right]=p$. This follows from Proposition 3.4 and basic properties of $p$-groups. Then $3\left(H_{i+1}\right) \supseteq 3\left(H_{i}\right)$ and

$$
\left[H_{i+1}: \mathcal{Z}\left(H_{i+1}\right)\right] \leqq\left[H_{i+1}: H_{i}\right]\left[H_{i}: \mathcal{Z}\left(H_{i}\right)\right] \leqq p p^{i(e+2)} \leqq p^{(i+1)(e+2)} .
$$

Now assume that $[G: \mathfrak{C}(A)]>p^{e}$. Since $\mathfrak{C}(A) \supseteqq H_{i}$ and $H_{i}$ has index $p^{4 e-i}$ we see that $[G: \subseteq(A)] \geqq p^{e+1}$. Then by Lemma 4.4 we can find an element $x \in G$ with $x \notin \subseteq(S)$ and hence $x \notin H_{i}$ with $\left[A: \widetilde{S}_{A}(x)\right] \leqq p^{e+1}$. Let $H_{i+1}$ be a subgroup with $\left.\left\langle H_{i}, x\right\rangle \supseteqq H_{i+1}\right\rangle H_{i}$ and $\left[H_{i+1}: H_{i}\right]=p$. Then $3\left(H_{i+1}\right) \supseteqq \mathfrak{S}_{A}(x)$ so

$$
\begin{aligned}
{\left[H_{i+1}: 3\left(H_{i+1}\right)\right] } & \leqq\left[H_{i+1}: H_{i}\right]\left[H_{i}: 3\left(H_{i}\right)\right]\left[3\left(H_{i}\right): \mathfrak{\complement}_{A}(x)\right] \\
& \leqq p p^{i(e+2)} p^{e+1}=p^{(i+1)(e+2)}
\end{aligned}
$$

and the induction follows.

With $i=3 e$ we have a subgroup $H=H_{3 e}$ of index $p^{e}$ with $[H: \mathfrak{3}(H)] \leqq p^{3 e(e+2)}$.

The bound given in the above theorem is certainly not best possible. However we can show by example that a result of this nature cannot be extended to subgroups of index $<p^{e}$. We first need another example of a group with r.x.1.

EXAMPLE 4.5. Let $A$ be an elementary abelian $p$-group with $p n$ generators. Let $F_{2}$ be the semi-direct product of $A$ by an element of order $p$ which permutes a basis of $A$ in cycles of length $p$. By Proposition 1.1, $F_{2}$ has r.x.1. Also $\left[F_{2}: 3\left(F_{2}\right)\right]=p p^{(p-1) n}$. Hence by choosing $n$ sufficiently large we can make this index as large as we please.

Now set $G=F_{2} \times F_{2} \times \cdots \times F_{2}$ the direct product of $e$ copies of $F_{2}$. Then $G$ has r.x.e. Any subgroup $H$ of $G$ of index $<p^{e}$ must project fully onto one of the direct summands. Hence there can be no bound on $[H: 3(H)]$.

The following two propositions are more general than we will need to study groups of r.x.1. However they are of interest in their own right.

Proposition 4.6. Let $G$ be an arbitrary group. Let $q$ be the smallest prime for which $\bigodot_{q}(G)$ is noncyclic. Given any $n$ nonidentity elements $g_{1}, \cdots, g_{n} \in G$ with $n \leqq q$ then $G$ has an absolutely irreducible representation $\mathfrak{r}$ with $\mathfrak{r}\left(g_{i}\right) \neq 1$ for $i=1,2, \cdots, n$. Moreover $n \leqq q$ is best possible. 
Proof. We first show this for abelian $G$ by induction on $|G|$. If $G$ is cyclic the result is of course clear. So assume that $G$ is not cyclic. Then $G$ has a subgroup of type $(q, q)$. This group has $q+1$ disjoint subgroups of order $q$. Since there are only $n \leqq q$ elements there is some subgroup $K$ of order $q$ not containing any of the $g_{i}$. Then $g_{i} \rightarrow \bar{g}_{i} \neq 1$ in $G / K$. If $\bar{q}$ is the smallest prime with $\Im_{\bar{q}}(G / K)$ noncyclic then $n \leqq q \leqq \bar{q}$. So by induction there is a linear character $\lambda$ of $G / K$ with $\lambda\left(\bar{g}_{i}\right) \neq 1$. View $\lambda$ as a character of $G$ and the result follows.

Now let $G$ be arbitrary but suppose that all the $g_{i}$ commute. Set $A=\left\langle g_{i} \mid i=1,2, \cdots, n\right\rangle$. Then $A$ is abelian and for any prime $p$, $\mathfrak{S}_{p}(A) \subseteq \mathfrak{S}_{p}(G)$ for some Sylow $p$-subgroup of $G$. Thus if $\widetilde{q}$ is the smallest prime with $\mathfrak{S}_{\tilde{q}}(A)$ noncyclic we have $n \leqq q \leqq \widetilde{q}$. Then by the previous result $A$ has a linear character $\lambda$ with $\lambda\left(g_{i}\right) \neq 1$ for all $i$. Let $\chi$ be a constituent of $\lambda^{*}$. The $\lambda$ is a constituent of $\chi \mid A$ so the $g_{i}$ are not in the kernel of $\chi$.

Finally let $G$ be arbitrary. We prove the result by induction on $n$. If all the $g_{i}$ commute and in particular if $n=1$ the result follows by the above. Suppose then that say $g_{n}$ and $g_{n-1}$ do not commute. Set $h=\left(g_{n}, g_{n-1}\right)$ so that $h \neq 1$. Then by induction we can find an absolutely irreducible representation $x$ of $G$ with $\mathfrak{x}\left(g_{i}\right) \neq 1$ for $i=$ $1,2, \cdots,(n-2)$ and $\mathfrak{x}(h) \neq 1$. But $\mathfrak{x}(h)=\left(\mathfrak{c}\left(g_{n}\right), \mathfrak{x}\left(g_{n-1}\right)\right) \neq 1$ so certainly $\mathfrak{r}\left(g_{n}\right) \neq 1$ and $\mathrm{r}\left(g_{n-1}\right) \neq 1$.

To see that $n \leqq q$ is best possible let $G$ be abelian of type $(q, q)$. If $g_{1}, \cdots, g_{q+1}$ are generators of the $q+1$ proper subgroups of $G$ then one is always in the kernel of every linear character. This completes the proof.

Proposition 4.7. Let $G$ be a group with r.x.e and suppose all primes $q$ dividing $|G|$ are larger than $e_{\text {o }}$ Let $A_{0}<A_{1}<\cdots<A_{m}$ be a series of normal abelian subgroups of $G$ such that

$$
\mathfrak{C}\left(A_{0}\right)>\mathfrak{C}\left(A_{1}\right)>\cdots>\mathfrak{S}\left(A_{m}\right) .
$$

Then $m \leqq e$.

Proof. It suffices to show that no such series exists with $m=$ $e+1$. So suppose $m=e+1$ in the above and set $N_{i}=\mathbb{C}\left(A_{i}\right)$. Then by assumption $N_{i}$ centralizes $A_{i}$ but not $A_{i+1}$ so we have $1 \neq\left(N_{i}, A_{i+1}\right)$. Choose $x_{i} \in\left(N_{i}, A_{i+1}\right)$ with $x_{i} \neq 1$ for $i=0,1, \cdots, e$. These are $e+1 \leqq q$ nonidentity elements of $G$ for all $q$ dividing $|G|$. Thus by Proposition 4.6, $G$ has an absolutely irreducible representation $r$ with $\mathrm{r}\left(x_{i}\right) \neq 1$ for $i=0,1, \cdots, e$.

Set $\bar{N}_{i}=\mathfrak{x}\left(N_{i}\right), \bar{A}_{i}=\mathfrak{x}\left(A_{i}\right)$ and since $A_{e+1}$ is abelian we can assume 
it is represented by diagonal matrices. Let $\mathscr{L}\left(\bar{A}_{i}\right)$ denote the linear subspace of matrices spanned by $\bar{A}_{i}$. If $\chi$ is the the character of $x$ with say $\chi \mid A_{i}=a_{i} \sum_{1}^{t i} \lambda_{j}$ since $A_{i} \Delta G$ then clearly $\operatorname{dim} \mathscr{L}\left(\bar{A}_{i}\right)=t_{i}$ which is a power of $p$ since $t_{i}$ divides the degree of $\mathrm{r}$. Set $t_{i}=p^{s_{i}}$.

Since $A_{i+1}>A_{i}$ we have $\mathscr{L}\left(\bar{A}_{i+1}\right) \supseteqq \mathscr{L}\left(\bar{A}_{i}\right)$. But since $\left(\bar{N}_{i}, \bar{A}_{i+1}\right) \neq 1$, $\bar{N}_{i}$ centralizes $\mathscr{L}\left(\bar{A}_{i}\right)$ but not $\mathscr{L}\left(\bar{A}_{i+1}\right)$. Hence $\mathscr{L}\left(\bar{A}_{i+1}\right)>\mathscr{S}\left(\bar{A}_{i}\right)$ and so $s_{i+1}>s_{i}$ for $i=0,1, \cdots, e$. Since $s_{0} \geqq 0$ we have $s_{e+1} \geqq e+1$. But $G$ has r.x.e so $\operatorname{deg} \mathrm{r} \leqq p^{e}$ and since $\mathscr{L}\left(\bar{A}_{e+1}\right)$ is a space of diagonal matrices $\operatorname{dim} \mathscr{L}\left(\bar{A}_{e+1}\right)=p^{s_{e+1}} \leqq p^{e}$, a contradiction. Thus the result follows.

THEOREM C 4.8. Group $G$ has r.x.1 if and only if $G$ is one of the following types:

(i) An abelian group

(ii) A group with a normal abelian subgroup of index $p$

(iii) A group with center of index $p^{3}$.

Proof. Let us assume that $G$ is a non-abelian group which has no normal abelian subgroup of index $p$. If $G$ has r.x.1 then by Lemma $3.9 G$ has a normal abelian subgroup $A$ of index $p^{2}$. Clearly $A=\mathbb{E}(A)$.

Let $K$ be a subgroup of $G$ with $G>K>A$. Since $G / A$ is of order $p^{2}, K$ is normal in $G$. Clearly $3(K)<A$. Set $A_{0}=3(G), A_{1}=$ $3(K)$ and $A_{2}=A$. Then $A_{0} \subseteq A_{1}<A_{2}$. Suppose that $A_{0} \neq A_{1}$. Then we have $A_{0}<A_{1}<A_{2}$ and

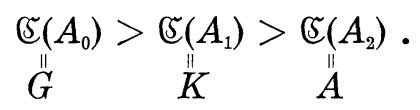

Clearly the hypotheses of Proposition 4.7 are satisfied by $G$ and so this cannot occur. Hence $3(K)=3(G)$.

Let $H=\$ 2(G)$. We show now that $H$ is central. In view of the above it suffices to show that $\mathfrak{S}(H)>A$. Let $P=\mathfrak{S}_{p}(A)$. Then $A=P \times H$ and $P \Delta G$. Now the group $\bar{G}=G / P$ has r.x.(1, 0$)$ since its Sylow $p$-subgroup has order $p^{2}$. Thus by Theorem $A, \bar{G}$ has an abelian subgroup of index $p$. Let $B$ be its complete inverse image in $G$. Then $B \supseteqq H$ but $B \nsubseteq A$. Choose $x \in B$ - $A$ with $x^{p} \in A$. Since $\bar{B}$ is abelian, $(x, H) \subseteq P$. But $H$ is normal so $(x, H) \subseteq H$. Hence $(x, H)=1$ and $H \subseteq 3(K)=3(G)$ where $K=\langle A, x\rangle$.

Thus $G=H \times \mathfrak{S}_{p}(G)$ and it suffices to assume that $G$ is a $p$-group. Now by Lemma 4.4 since $[G: A]=p^{2}$ there is an element $x \notin \Subset(A)=A$ with $\left[A: \mathbb{S}_{A}(x)\right]=p$. We can assume that $x^{p} \in A$. Then with $K=$ $\langle A, x\rangle$ we have $[A: \mathfrak{Z}(K)]=[A: \mathfrak{Z}(G)]=p$. Hence $[G: \mathfrak{Z}(G)]=p^{3}$.

Conversely by Propositions 1.1 and 1.4 any group of type (i), (ii) or (iii) has r.x.l. 
That all of the above possibilities occur can be seen by examples. Type (i) is clear. Group $F_{2}$ is an example of type (ii) which is neither (i) nor (iii). Finally $F_{1}$ is type (iii) but not (i) or (ii).

5. Groups with r.b.n. The results of the previous sections apply to rather special groups. We consider the more general case now.

DeFinition 5.1. We say group $G$ has r.b.n (representation bound $n$ ) if the degrees of all the absolutely irreducible characters of $G$ are $\leqq n$.

Let $h_{1}$ be the function with the following property. If $G$ has r.b.n then $G$ has a subinvariant abelian subgroup whose index is at most $h_{1}(n)$. Moreover $h_{1}$ is the smallest such function.

Let $h_{2}$ (respectively $h_{3}$ ) be the corresponding function where we restrict $G$ to the class of solvable (respectively nilpotent) groups.

We show here that the $h_{i}$ exist and obtain bounds for each. This may be viewed as an analog of Jordan's Theorem ([14] Satz 200) which we state below.

Proposition 5.2. (Jordan). There exists a function $J$ with the following property. If $G$ is a complex linear group of degree $n$ then $G$ has a normal abelian subgroup with index $\leqq J(n)$.

We assume that the function $J$ above is best possible. One of the known bounds for $J$ is

$$
J(n) \leqq n ! 12^{n(\pi(n+1)+1)}
$$

where $\pi(n+1)$ is the number of primes $\leqq n+1$.

We prove

THEOREM D 5.3. $\quad h_{1}(n) \leqq J(2 n)$

$$
\begin{aligned}
& h_{2}(n) \leqq n^{3 / 2 \log _{2} 2 n} \\
& h_{3}(n) \leqq n^{4} .
\end{aligned}
$$

The third case is an immediate corollary of Theorem A. Let $G$ be nilpotent so that $G=P_{1} \times P_{2} \cdots \times P_{r}$ where $P_{i}$ is a Sylow $p_{i^{-}}$ subgroup of $G$. Suppose the degree of the biggest character of $P_{i}$ is $p_{i}^{e_{i}}$. Then the biggest character of $G$ has degree $\Pi_{i} p_{i}^{e_{i}} \leqq n$. Now by Theorem $\mathrm{A}, P_{i}$ has a subinvariant abelian subgroup $A_{i}$ with $\left[P_{i}: A_{i}\right] \leqq p_{i}^{4 e_{i}}$. If $A=A_{1} \times A_{2} \times \cdots \times A_{r}$ then $A$ is a subinvariant abelian subgroup of $G$ with index $\leqq \Pi p_{\imath}^{4 e} i \leqq n^{4}$. Hence we need only 
consider the first two cases.

We use a variant on the techniques of $\S 2$. We first need a few simple facts about the function $J$.

Lemma 5.4. For all $n \geqq 1, J(n) \geqq n^{3}$. For all $n_{1}, n_{2}$ we have $J\left(n_{1}\right) J\left(n_{2}\right) \leqq J\left(n_{1}+n_{2}\right)$.

Proof. The symmetric group $S_{n+1}$ has a faithful irreducible character of degree $n$. Since for $n \geqq 4, S_{n+1}$ has no normal abelian subgroup we have $J(n) \geqq(n+1)$ ! for $n \geqq 4$. Eut then

$$
J(n) \geqq(n+1) ! \geqq(n+1) n(n-1) 2=2 n\left(n^{2}-1\right) \geqq n^{3} .
$$

Thus we need only consider $n=1,2,3$. Case $n=1$ is clear.

Let $H$ be a linear group of degree 2. Then $H / 3(H)$ is a collineation group of degree two. These are known to be ([12] Chapter $\mathrm{X}$ ) (1) cyclic or dihedral, (2) order $<60$ and (3) the alternating group $A_{5}$ of order 60. From this we conclude immediately that $J(2)=60$. Hence $60=J(2)>2^{3}$ and $J(3) \geqq J(2)=60>3^{3}$. The first result follows.

Let $G_{\imath}$ be a linear group of degree $n_{i}$ having no normal abelian subgroup of index less than $J\left(n_{i}\right)$ for $i=1,2$. Then $G=G_{1} \times G_{2}$ is a linear group of degree $n_{1}+n_{2}$ having no rormal abelian subgroup of index less than $J\left(n_{1}\right) J\left(n_{2}\right)$. Hence $J\left(n_{1}+n_{2}\right) \geqq J\left(n_{1}\right) J\left(n_{2}\right)$ and the result follows.

Lemma 5.5. Given $K \Delta G$ with $G / K$ non-abelian. If $G$ has r.b.n then $K$ has r.b.(n/2).

Proof. Let $\varphi$ be an absolutely irreducible character of $K$ and let $\chi$ be a constituent of $\varphi^{*}$. Set $\chi \mid K=\psi$. Then $\psi=a \Sigma_{1}^{t} \varphi_{i}$ with $\varphi_{1}=\varphi$. Now $\psi^{*}=[G: K] \chi$ on $K$ and $\psi^{*}=0$ off $K$. Hence if $\rho$ denotes the character of the regular representation of $G / K$ in $G$ we have $\psi^{*}=\chi \rho_{\text {. }}$ If $\left\{\beta_{i}\right\}$ is the set of absolutely irreducible characters of $G / K$ in $G$ with $b_{i}=\operatorname{deg} \beta_{i}$ then $\rho=\Sigma b_{i} \beta_{i}$.

We compute $\left[\psi^{*}, \psi^{*}\right]$. By Frobenius reciprocity this is equal to

$$
\left[\psi, \psi^{*} \mid K\right]_{K}=[G: K][\psi, \psi]_{K} .
$$

On the other hand $w^{*}=\chi \rho=\Sigma b_{i} \chi \beta_{i}$ so we have

$$
[G: K][\psi, \psi]_{K}=\left[\psi^{*}, \psi^{*}\right]=\Sigma_{i j} b_{i} b_{\jmath}\left[\chi \beta_{i}, \chi \beta_{j}\right] \text {. }
$$

Suppose for some nonlinear character $\beta=\beta_{i}$ we have that $\chi \beta$ is irreducible. Then

$$
n \geqq \operatorname{deg} \chi \beta=(\operatorname{deg} \chi)(\operatorname{deg} \beta) \geqq 2 \operatorname{deg} \chi \text {. }
$$


So $\operatorname{deg} \chi \leqq n / 2$. But $\operatorname{deg} \varphi \leqq \operatorname{deg} \chi$ so the result follows here.

If for some nonlinear $\beta=\beta_{i}$ we have that $\chi \beta$ is reducible then $[\chi \beta, \chi \beta]>1$. Hence

$$
[G: K][\psi, \psi]_{K}>\Sigma b_{i}^{2}=[G: K]
$$

so that $[\psi, \psi]_{\kappa}>1$. Now $[\psi, \psi]_{K}=a^{2} t>1$ implies that $a t \geqq 2$. But

$$
n \geqq \operatorname{deg} \chi=a t \operatorname{deg} \varphi \geqq 2 \operatorname{deg} \varphi
$$

so $n / 2 \geqq \operatorname{deg} \varphi$ in this case also. Hence $K$ has r.b. $(n / 2)$ and the result follows.

We are now ready to prove the theorem.

Proof of Theorem $D$. The proof is by induction on $|G|$. If $G$ is abelian the result is trivial so we assume that $G$ is non-abelian. Choose $K \Delta G$ maximal with respect to $G / K$ being non-abelian. If $E=G / K$ has a nontrivial normal abelian subgroup then $E$ is extraspecial and we have two possibilities by Proposition 2.2. Thus there are three cases to consider: Case $P$, Case $Q$ and Case $R$, the latter being the possibility that $E$ has no nontrivial normal abelian subgroup. Note Case $R$ cannot occur if $G$ is solvable.

We point out the obvious fact that the r.b.n property (as well as solvability) is inherited by subgroups and quotient groups.

Case P: Let $\varphi$ be a character of $Z$ as in Lemma 3.5. Then

$$
(\operatorname{deg} \varphi)^{2} \leqq 1 / p^{m} t(\operatorname{deg} \chi)\left(\operatorname{deg} \chi_{1}\right)
$$

so $\operatorname{deg} \varphi$ is properly smaller than at least one of $\operatorname{deg} \chi$ and $\operatorname{deg} \chi_{1}$. Since $\operatorname{deg} \varphi$ divides both $\operatorname{deg} \chi$ and $\operatorname{deg} \chi_{1}$ we have, if say $\operatorname{deg} \varphi<\operatorname{deg} \chi$, that $\operatorname{deg} \varphi \leqq 1 / 2 \operatorname{deg} \chi \leqq n / 2$. Thus $Z$ has r.b.(n/2). Now $[G: Z]=$ $p^{2 m} \leqq n^{2}$ since $p^{m}$ is the degree of a character of $G$. Thus

$\left(^{*}\right) \quad G$ has a normal subgroup $Z$ of index $\leqq n^{2}$ with r.b. $(n / 2)$.

Case $Q$ : Let $Q$ be the inverse image in $G$ of $E^{\prime}$ the Sylow $q$ subgroup of $E$. Now $[G: Q]$ is the degree of a character of $G$ by (vi) of Proposition 2.2. Hence $[G: Q] \leqq n$. If $Q$ has r.b. $(n / 2)$ then

(**) $G$ has a normal subgroup $Q$ of index $\leqq n$ with r.b. $(n / 2)$.

We assume now that this is not the case. Let $\theta$ be a character of $Q$ of degree $>n / 2$. If $\chi$ is a constituent of $\theta^{*}$ then $\chi \mid Q=a \Sigma_{1}^{t} \theta_{i}$ and $\operatorname{deg} \chi=a t \operatorname{deg} \theta$. But $\operatorname{deg} \chi \leqq n$ so $a=t=1$. Hence for all $x \in G-Q$ we have $\theta^{x}=\theta$. Let $\mu$ be any linear character of $Q$ 
corresponding to one of $E^{\prime}$. Then $\theta \mu$ has degree $>n / 2$ so also $(\theta \mu)^{x}=$ $\theta \mu$. Thus

$$
\theta \mu=(\theta \mu)^{x}=\theta^{x} \mu^{x}=\theta \mu^{x}
$$

and $\theta=\theta \mu^{x} \bar{\mu}$. Hence $\theta$ vanishes off the kernel of $\mu^{x} \bar{\mu}$. Now given any linear character $\lambda$ of $Q / K$ we can find a character $\mu$ with $\lambda=\mu^{x} \bar{\mu}$ by (vii) of Proposition 2.2. Hence $\theta$ vanishes off the kernel of $\lambda$. But the intersection of all these kernels is $K$ and so $\theta$ vanishes off $K$.

Let $\theta \mid K=a \Sigma_{1}^{t} \varphi_{i}$. Then $[\theta|K, \theta| K]_{K}=a^{2} t$. On the other hand since $\theta$ vanishes off $K$

$$
1=[\theta, \theta]_{Q}=(|K| /|Q|)[\theta|K, \theta| K]_{K}=(|K| /|Q|) a^{2} t .
$$

Hence

$$
[Q: K]=a^{2} t \leqq\left(a t \operatorname{deg} \varphi_{1}\right)^{2}=(\operatorname{deg} \theta)^{2} \leqq n^{2} .
$$

So $[G: K]=[G: Q][Q: K] \leqq n^{3}$. Since $G / K$ is non-abelian, $K$ has r.b. $(n / 2)$ by Lemma 5.5 so we have

$\left.{ }^{* * *}\right) \quad G$ has a normal subgroup $K$ of index $\leqq n^{3}$ with r.b. $(n / 2)$.

Case $R$ : Suppose $E=G / K$ has no normal abelian subgroup. Let $\chi$ be a nonlinear character of $E$ with kernel $N$. If $N>1$ then $N \supseteqq E^{\prime}$ and so $\chi$ would be linear. Hence $N=1$ so $E$ is a faithful linear group of degree $m \leqq n$ since $G$ has r.b.n. But then $E$ has a normal abelian subgroup of index $\leqq J(m) \leqq J(n)$ by Proposition 5.2. Hence $[G: K] \leqq J(n)$. Since $E$ is non-abelian, $K$ has r.b. $(n / 2)$ by Lemma 5.5. Thus

$(* * *) \quad G$ has a normal subgroup $K$ of index $\leqq J(n)$ with r.b.(n/2).

We now apply induction. If $G$ is given to be solvable then so is every quotient and subgroup. Thus only Cases $P$ and $Q$ occur. Then $(*),\left({ }^{*}\right)$, and $\left({ }^{* *}\right)$ imply that $G$ has a normal subgroup $N$ of index $\leqq n^{3}$ and with r.b. $(n / 2)$. By induction $N$ has subinvariant abelian subgroup $A$ with

$$
[N: A] \leqq(n / 2)^{3 / 2} \log _{2} n
$$

Then $A$ is subinvariant in $G$ with

$$
[G: A] \leqq n^{3}(n / 2)^{3 / 2 \log _{2} n}=n^{3 / 2 \log _{2} 2 n}
$$

and the bound for $h_{2}$ follows.

In the general case all four possibilities can occur. Since by Lemma 5.4, $J(n) \geqq n^{3}$ we see that $G$ has a normal subgroup $N$ of 
index $\leqq J(n)$ with r.b. $(n / 2)$. By induction $N$ has a subinvariant abelian subgroup $A$ with $[N: A] \leqq J(n)$. Then $A$ is subinvariant in $G$ with

$$
[G: A]=[G: N][N: A] \leqq J(n) J(n) \leqq J(2 n)
$$

by Lemma 5.4 again. This completes the proof.

6. Normal Sylow $p$-subgroups. The analogy between groups with r.b.n and linear groups of degree $n$ can be carried further. We show

Theorem E 6.1 Let $G$ be a group with r.b.n. If $p$ is a prime with $p>n$ then $\mathfrak{S}_{p}(G)$ is normal and abelian.

If $n=1$ the result is trivial. Thus we assume that $n \geqq 2$ and hence $p>2$. Let $G$ be a group of order $g=p^{a} g_{0}$ with $g_{0}$ prime to $p$ and $a \geqq 1$. Let $\delta=\varepsilon \delta_{0}$ be a primitive $g$ th root of unity over the rational numbers $\boldsymbol{Q}$ with $\in$ a primitive $p^{a}$ th root and $\delta_{0}$ a $g_{0}$ th root. Then as is well known, the Galois group of $\boldsymbol{Q}(\delta) / \boldsymbol{Q}\left(\delta_{0}\right)$ is cyclic of order $p^{a-1}(p-1)$. Let $\sigma$ be an element of order $p-1$ in this group. Then $\sigma$ is an automorphism of $\boldsymbol{Q}(\delta)$ which fixes $\boldsymbol{Q}\left(\delta_{0}\right)$ and cyclically permutes the $p-1$ primitive $p$ th roots of unity. Moreover as is well known $\sigma$ permutes the characters of $G$.

Lemma 6.2. Let $G$ be a faithful linear group with character $\chi$ such that $\chi$ is fixed under the action of $\sigma$. Then $\operatorname{deg} \chi \geqq p-1$. If $\operatorname{deg} \chi=p-1$ and $G$ has trivial center then $G$ has no elements of order $p q$ with $q$ prime to $p$.

Proof. Let $x$ be an element of order $p$ in $G$. Its eigenvalues (in the matrix representation) are all $p$ th roots of unity. Since $\chi^{\sigma}(x)=$ $\chi(x)$ we see that $\chi(x)$ is rational. From the form of the minimal polynomial of a primitive $p$ th $\operatorname{root} \mu$ over $\boldsymbol{Q}$ we see that $x$ has at least $p-1$ eigenvalues. Hence $\operatorname{deg} \chi \geqq p-1$.

If $\operatorname{deg} \chi=p-1$ then the eigenvalues of $x$ are $\mu, \mu^{2}, \cdots, \mu^{p-1}$. Clearly we can assume that $x$ is in diagonal form so that

$$
x=\operatorname{diag}\left(\mu, \mu^{2}, \cdots, \mu^{p-1}\right) \text {. }
$$

Let $y$ be an element of $G$ of order $q$ where $q$ is prime to $p$. Suppose $x y=y x$. . Since all the diagonal entries of $x$ are distinct, $y$ is also diagonal. Say $y=\operatorname{diag}\left(\lambda_{1}, \lambda_{2}, \cdots, \lambda_{p_{-1}}\right)$ and so each $\lambda_{i}$ is a $q$ th root of unity. Thus

$$
\Sigma_{i} \lambda_{i} \mu^{i}=\chi(x y)=\chi^{\sigma}(x y)=\Sigma_{i} \sigma\left(\lambda_{i} \mu^{i}\right)=\Sigma_{i} \lambda_{i} \sigma\left(\mu^{i}\right)
$$

since $\sigma$ fixes $\boldsymbol{Q}\left(\delta_{0}\right)$. 
Suppose $\sigma(\mu)=\mu^{s}$. Then with the subscripts taken modulo $p$ we have

$$
\Sigma_{i} \lambda_{i s} \mu^{i s}=\Sigma_{i} \lambda_{i} \mu^{i}=\Sigma_{i} \lambda_{i} \sigma\left(\mu^{i}\right)=\Sigma_{i} \lambda_{i} \mu^{i s}
$$

or

$$
\Sigma_{i}\left(\lambda_{i}-\lambda_{i s}\right) \mu^{i s}=0 .
$$

Since the $\mu^{i s}$ are linearly independent over $\boldsymbol{Q}\left(\delta_{0}\right)$ we have $\lambda_{i}=\lambda_{i s}$ for each $i$. Hence $\lambda_{1}=\lambda_{s}=\lambda_{s^{2}}=\cdots$ and all of the $\lambda_{i}$ are equal since $\sigma$ has order $p-1$. Thus $y$ is central.

Suppose $z$ is an element of $G$ of order $p q$. Then $x=z^{q}$ has order $p$ and $y=z^{p}$ has order $q$. Moreover $x$ and $y$ commute. By the above $y \in 3(G)=1$, a contradiction. Thus the lemma is proved.

Suppose $\sigma(\delta)=\delta^{u}$. Since $\sigma$ is an automorphism $u$ is prime to $g=|G|$. Now $\sigma$ fixes $Q\left(\delta_{0}\right)$ so we see that $u \equiv 1 \bmod g_{0}$. Let $\alpha$ be the permutation on the elements of $G$ defined by $\alpha(x)=x^{u}$. If $x$ is $p$-regular $\left(x^{g_{0}}=1\right)$ then $\alpha(x)=x$. Clearly $\alpha$ permutes the conjugacy classes of $G$. If $\chi$ is any character of $G$ then $\chi^{\sigma}(x)=\chi(\alpha(x))$. It follows ([3], $\S 6$ and 7) that the number of irreducible characters fixed by $\sigma$ is equal to the number of classes fixed by $\alpha$. Since both $\sigma$ and $\alpha$ have order $p-1$, the number of transitivity classes in the respective actions of groups $\langle\sigma\rangle$ and $\langle\alpha\rangle$ on the characters and classes are equal.

Lemma 6.3. Let $G$ have r.b. $(p-1)$. Suppose $G^{\prime}>1$ is the unique normal subgroup of $G$ and assume that $\left[G: G^{\prime}\right]$ divides $p$. Then $p$ is prime to $|G|$.

Proof. Suppose to the contrary that $|G|=g=p^{a} g_{0}$. Now $G$ has $t=\left[G: G^{\prime}\right]$ linear characters and clearly all nonlinear characters are faithful. If all nonlinear characters of $G$ have degree $p-1$ we have

$$
g=t+b(p-1)^{2} .
$$

But $p-1$ being the degree of an irreducible character must divide $g$. Thus $p-1$ divides $t=1$ or $p$, a contradiction for $p>2$.

Since $G$ has r.b. $(p-1)$ some nonlinear faithful character of $G$ has degree $k<p-1$. Thus by Feit's theorem ([6]), $\left[G: \mathfrak{O}_{p}(G)\right]$ is divisible by $p$ to at most the first power. Here $\mathfrak{D}_{p}(G)$ is the maximal normal $p$-subgroup of $G$. Since $G$ is not a $p$-group (having r.b. $(p-1)$ it would be abelian) we see that $\mathfrak{S}_{p}(G)=1$. Hence $p$ divides $|G|$ to the first power only and so $\widetilde{\subseteq}_{p}(G)$ is cyclic of order $p$.

Since $G$ has at least two $p$-regular classes, including the identity class, $\alpha$ fixes at least two classes and hence $\sigma$ fixes at least two charac- 
ters. Now $\sigma$ fixes the principal character but no other linear character since $\left[G: G^{\prime}\right]=1$ or $p$. Hence $\sigma$ fixes a nonlinear faithful irreducible character $\chi$ and Lemma 6.2 applies. Since $G$ has r.b. $(p-1), \chi$ has degree $p-1$ and thus $G$ has no elements of order $p q$ with $q$ prime to $p$. Note $3(G)=1$ since otherwise $G$ would be a cyclic extension of $G^{\prime} \subseteq 3(G)$ and hence would be abelian.

This implies that every $p$-singular (order divisible by $p$ ) class consists of elements of order $p$. Thus if $x$ and $y$ are $p$-singular elements of $G$ then $x$ is conjugate to some power of $y$ by the second Sylow theorem. Hence all $p$-singular classes form a single transitivity class under the action of $\langle\alpha\rangle$. We conclude then that the total number of $\langle\alpha\rangle$-transitivity classes is equal to the number of $\alpha$-fixed classes plus one and the same is therefore true for $\sigma$. Thus all irreducible characters moved by $\sigma$ are $\langle\sigma\rangle$-conjugate. Therefore their number $r>1$ divides $p-1$ and their degrees are all equal to $k$. Hence we have

$$
g=t+r k^{2}+b(p-1)^{2} .
$$

Since $r$ divides $p-1$ and $p-1$ divides $g$ we see that $r$ divides $t$. But $t=1$ or $p$ so $r=1$, a contradiction, and the result follows.

Proof of Theorem $E$. If $G$ has a normal Sylow $p$-subgroup then the latter is clearly abelian. We show by induction on $|G|$ that $\mathfrak{S}_{p}(G) \Delta G$.

We can clearly assume that $p$ divides $|G|$ and that $G$ is nonabelian. By Lemma 6.3, $G$ has a normal subgroup $N$ with $G>N>1$ and $[G: N] \neq p$. Suppose that $p$ divides $|G / N|$. Now $G / N$ has r.b.n so $G / N$ has a normal Sylow $p$-subgroup by induction. If $G / N$ is not $p$-group then $M=N \Im_{p}(G / N)$ is normal in $G, G>M>1$ and $p$ divides $|M|$. If $G / N$ is a $p$-group then since $|G / N| \neq p$ we can again find such a normal subgroup $M$. If $p$ does not divide $|G / N|$ then $p$ divides $|N|$. Hence $G$ has a proper normal subgroup $M$ with $p$ dividing $|M|$. Now $G>M$ so by induction $\mathfrak{S}_{p}(M)$ is normal in $M$. Since $\mathfrak{S}_{p}(M)$ is then in fact characteristic, it is normal in $G$. Set $K=G / \mathfrak{S}_{p}(M)$. Then $|K|<|G|$ so $K$ has a normal Sylow $p$-subgroup. Hence $\mathfrak{S}_{p}(G)=$ $\mathfrak{S}_{p}(M) \mathfrak{S}_{p}(K)$ is normal in $G$. This completes the proof.

7. Infinite groups with r.b.n. We show here that Theorem D holds for arbitrary (not necessarily finite) discrete groups.

DeFinition 7.1. Let $G$ be an arbitrary discrete group and let $C[G]$ be its group algebra over the complex numbers. If $\mathscr{F}$ is an irreducible representation of the algebra then $\mathscr{F}(\boldsymbol{C}[G])=P$ is primitive and hence is isomorphic to a dense set of linear transformations 
over $D$, the commuting ring of $\mathscr{F}$ ([10] page 28). Let $L$ be the center of $D$. If $\operatorname{dim}_{L} P<\infty$ then we say $\mathscr{F}$ is finite and since $P$. is central simple over $L$ ([10] page 122) we have $\operatorname{dim}_{L} P=m^{2}$. We set $m=\operatorname{deg} \mathscr{F}$, the degree of $\mathscr{F}$.

If $G$ is finite then $C$ is always the commuting ring of every irreducible representation. Thus the degree defined above agrees with the usual one in this case.

We say $G$ has r.b.n if all irreducible representations $\mathscr{F}$ of $C[G]$ are finite and for each $\operatorname{deg} \mathscr{F} \leqq n$.

The following lemma was obvious for finite groups.

LEMMA 7.2. If $G$ has r.b.n then so does every subgroup and quotient group.

Proof. The fact for quotient groups is clear. Now since $C[G]$ is semi-simple ([13] Theorem 5.2), a result of Amitsur ([1] Theorem 1) yields the fact that $G$ has r.b.n if and only if $C[G]$ satisfies the polynomial identity $\left[x_{1}, x_{2}, \cdots, x_{2 n}\right]=0$. But if $H$ is a subgroup of $G$ then $C[G] \supseteqq C[H]$ and so the result follows.

Proposition 7.3. A finitely generated linear group is a subdirect product of finite groups.

Proof. Let $G \leqq[R]_{m}$ the ring of $m \times m$ matrices over field $R$. Let $S$ be the prime field contained in $R$. Then $S=\boldsymbol{Q}$ if $R$ has characteristic zero and $S=G F(p)$ if $R$ has characteristic $p$. Let $g_{1}, g_{2}, \cdots, g_{r}$ generate $G$ as a semi-group, that is we include with each generator of $G$ its inverse. Each $g_{i}$ is represented as a matrix over $R$. Let $X$ denote the set of all the matrix entries for all the $g_{i}$. This is of course a finite set.

Given nonidentity $g \in G$ we must show that $G$ has a normal subgroup $N$ of finite index with $g \notin N$. Since $g \neq 1$ choose $x$ to be a nonzero entry of $g-1$ (subtraction in $[R]_{m}$ ). Now $X \cup\{x\}$ is a finite set so by the Extension Theorem for Places ([2] Contention, page 49) there exists a place $\phi$ of $R$ into $\bar{S}$, the algebraic closure of $S$, union $\infty$, the ideal point, with $\phi(X \cup\{x\}) \subseteq \bar{S}$ and $\phi(x) \neq 0$. Now $\phi$ clearly induces a homomorphism of $G$ into $[\bar{S}]_{m}$ with $g$ not in the kernel. Since $G$ is finitely generated it is clear that the image of $G$ is in fact contained in $[T]_{m}$ where $T$ is a finite algebraic extension of $S$. If $S=G F(p)$ then $T$ is a finite field and so the image of $G$ is a finite group. Hence if $N$ denotes the kernel of the homomorphism we have $[G: N]<\infty$ and $g \notin N$. Thus the result follows here.

Hence it suffices to assume that $S=\boldsymbol{Q}$. Then $T$ is a finite alge- 
braic extension of $\boldsymbol{Q}$ and hence $\operatorname{Int}(T)$, the ring of algebraic integers in $T$, is a Dedekind domain. Now every element of $T$ is of the form $\alpha / n$ where $\alpha \in \operatorname{Int}(T)$ and $n$ is a rational integer. Let $\pi$ denote the set of primes in the rational integers which divide the denominators of the $\phi(X)$ which of course generate all of the entries of $\phi(G)$ as a ring. Here we have let $\phi$ denote the natural extension of the place to the group homomorphism. Now $\pi$ is clearly a finite set. Let $\phi(x)=\alpha / n$ so $\alpha \neq 0$. Then $\alpha$ is contained in only a finite number of prime ideals of $\operatorname{Int}(T)$. Thus we can find a prime ideal $\mathfrak{p}$ of $\operatorname{Int}(T)$ with $\alpha \notin \mathfrak{p}$ and such that $\mathfrak{p}$ does not divide any of the primes in $\pi$. Then clearly $\phi(G) \subseteq[U]_{m}$ where $U=\operatorname{Int}(T)_{\mathfrak{p}}$ is $\operatorname{Int}(T)$ localized at $\mathfrak{p}$. The map $\theta: U \rightarrow U / p U$ induces a homomorphism of $\phi(G)$ whose image is finite since $U / \mathfrak{p} U$ is a finite field. But by choice of $\mathfrak{p}, \phi(x)$ is not in the kernel of $\theta$. Thus $\theta \phi$ is a homorphism of $G$ into a finite group with $g$ not in the kernel. This completes the proof.

We will have need for the following lemma of M. Hall ([11] page $56)$ : If $G$ is a finitely generated group then $G$ has only a finite number of subgroups of any given finite index. The following proposition was proved in [8]. We offer a shorter proof here due to G. Glauberman.

Proposition 7.4. Let $G$ be an arbitrary group. Then $G$ has an abelian subgroup with index at most $m$ if and only if every finitely generated subgroup of $G$ has such an abelian subgroup.

Proof. If $A$ is abelian with $[G: A] \leqq m$ then for any subgroup $H$ of $G$ we have

$$
m \geqq[G: A] \geqq[G \cap H: A \cap H]=[H: A \cap H] .
$$

Hence $A \cap H$ is an abelian subgroup of $H$ with index at most $m$.

Conversely let us assume that every finitely generated subgroup of $G$ has an abelian subgroup of index at most $m$. For each finite subset $\alpha$ of $G$ let $G_{\alpha}=\langle\alpha\rangle$ be the group generated by the elements in $\alpha$. Let $\mathscr{A}_{\alpha}$ be the set of abelian subgroups of $G_{\alpha}$ and let $m_{\alpha}=$ $\min \left\{\left[G_{\alpha}: A\right] \mid A \in \mathscr{A}_{\alpha}\right\}$. By assumption $1 \leqq m_{\alpha} \leqq m$ for each $\alpha$. Choose $\alpha_{0}$ such that $m_{0}=m_{\alpha_{0}}$ is the largest of the $m_{\alpha}$ 's and set $G_{0}=G_{\alpha_{0}}$.

Let $A_{1}, A_{2}, \cdots, A_{r}$ be the abelian subgroups of $G_{\alpha_{0}}$ having index $m_{0}$ in $G_{\alpha_{0}}$. By the above mentioned lemma of M. Hall there are only a finite number of these. We show that for some $i=1,2, \cdots$, or $r$ both $\left[G: \mathfrak{C}\left(A_{i}\right)\right] \leqq m_{0}$ and $\mathbb{C}\left(A_{i}\right)$ is abelian. This will of course yields the result. Suppose not. Then for each $i$ choose $\alpha_{i}$ to consist of two noncommuting elements of $\mathfrak{E}\left(A_{i}\right)$ if the latter is non-abelian or $m_{0}+1$ elements in distinct right cosets of $\subseteq\left(A_{i}\right)$ if $\left[G: \subseteq\left(A_{i}\right)\right]>m_{0}$. Let 
$\alpha=\alpha_{0} \cup \alpha_{1} \cup \cdots \cup \alpha_{r}$. This is a finite set so let $A_{\alpha}$ be an abelian subgroup of $G_{\alpha}$ with $\left[G_{\alpha}: A_{\alpha}\right]=m_{\alpha}$. Now

$$
m_{0} \geqq m_{\alpha}=\left[G_{\alpha}: A_{\alpha}\right] \geqq\left[G_{\alpha} \cap G_{0}: A_{\alpha} \cap G_{0}\right]=\left[G_{0}: A_{\alpha} \cap G_{0}\right] \geqq m_{0} .
$$

The last inequality follows since $A_{\alpha} \cap G_{0}$ is an abelian subgroup of $G_{0}$. Thus $\left[G_{0}: A_{\alpha} \cap G_{0}\right]=m_{0}=m_{\alpha}$ and $A_{\alpha} \cap G_{0}=A_{i}$ for some $i$. Say $A_{\alpha} \cap G_{0}=A_{1}$.

Since $A_{\alpha}$ is abelian we have $A_{\alpha} \subseteq \Im_{\theta_{\alpha}}\left(A_{1}\right)$. On the other hand

$$
\left[G_{\alpha}: \mathfrak{夭}_{G_{\alpha}}\left(A_{1}\right)\right] \geqq\left[G_{\alpha} \cap G_{0}: \mathfrak{\Im}_{G_{\alpha}}\left(A_{1}\right) \cap G_{0}\right]=\left[G_{0}: A_{1}\right]=m_{0}=m_{\alpha}
$$

since $A_{1}$ is its own centralizer in $G_{0}$. Thus $A_{\alpha}=\mathfrak{夭}_{G_{\alpha}}\left(A_{1}\right)$. Now $\alpha_{1} \subseteq G_{\alpha}$. Hence if $\subseteq\left(A_{1}\right)$ were non-abelian then $\alpha_{1}$ would contain noncommuting elements in $\widetilde{夭}_{G_{\alpha}}\left(A_{1}\right)=A_{\alpha}$. But $A_{\alpha}$ is abelian so this is not the case. On the other hand if $\left[G: \mathfrak{C}\left(A_{1}\right)\right]>m_{0}$ then $G_{\alpha}$ would contain $m_{0}+1$ elements in different right cosets of $\mathfrak{E}\left(A_{1}\right)$ and hence of $\mathfrak{E}_{G_{\alpha}}\left(A_{1}\right)=A_{\alpha}$. But $\left[G_{\alpha}: A_{\alpha}\right]=m_{0}$ so we have a contradiction here and the result follows.

We now consider the extension of Theorem D to arbitrary groups.

Definition 7.5. Let $\tilde{h}_{1}$ be the function with the following property. If $G$ is a not necessarily finite, discrete group with r.b.n then $G$ has a subinvariant abelian subgroup $A$ with $[G: A] \leqq \widetilde{h}_{1}(n)$. Moreover $\widetilde{h}_{1}$ is the smallest such function.

Let $\widetilde{h}_{2}$ (respectively $\widetilde{h}_{3}$ ) be the corresponding functions when we restrict $G$ to the class of solvable (respectively nilpotent) groups.

THEOREM F 7.6. The functions $\widetilde{h}_{i}$ are finite valued. In fact $\tilde{h}_{i}(n)=h_{i}(n)$ for $i=1,2,3$.

Proof. First we notice that the property of being solvable or nilpotent is inherited by subgroups and quotient groups. Let $\widetilde{h}$ be the function of interest here and let $h$ be the corresponding function for finite groups. Clearly $\widetilde{h}(n) \geqq h(n)$.

Let $G$ have r.b.n. If $F$ is a finitely generated subgroup of $G$ then by Lemma 7.2, $F$ has r.b.n. If we know that $F$ has an abelian subgroup to index $\leqq h(n)$ for all such $F$ then the result follows by Proposition 7.4. Hence it suffices to assume that $G$ is finitely generated.

Let $G$ be a finitely generated group with r.b.n. Since $C[G]$ is semi-simple, $G$ is clearly a subdirect product of the groups $\mathscr{F}(G)$ for all irreducible representations $\mathscr{F}$ of the algebra. But $\mathscr{F}$ is finite so $\mathscr{F}(G)$ is a finitely generated linear group. By Proposition 7.3, $\mathscr{F}(G)$ and hence $G$ is a subdirect product of finite groups. 
Now let $A_{1}, A_{2}, \cdots, A_{r}$ be the subgroups of $G$ of index $\leqq h(n)$. By M. Hall's lemma there are only a finite number of these. We show that for some $i, A_{i}$ is abelian. Suppose not. Choose nonidentity $x_{i} \in A_{i}^{\prime}$, the commutator subgroup of $A_{i}$, for $i=1,2, \cdots, r$. Since $G$ is a subdirect product of finite groups we can find a normal subgroup $N$ of $G$ with $[G: N]<\infty$ and $x_{i} \notin N$ for all $i$. Now $G / N$ is a finite group with r.b.n and hence $G / N$ has an abelian subgroup of index $\leqq h(n)$. Let $B$ be its complete inverse image in $G$. Then $[G: B] \leqq h(n)$ so $B=A_{i}$ for some $i$. Say $B=A_{1}$. Now $B / N$ is abelian so $B^{\prime} \subseteq N$. But by choice of $N, A_{1}^{\prime} \nsubseteq N$. This is the required contradiction. Thus $G$ has an abelian subgroup of index $\leqq h(n)$ and hence $\widetilde{h}(n) \leqq h(n)$ and the result follows.

\section{REFERENCES}

1. S. A. Amitsur, Groups with representations of bounded degree II, Illinois J. Math. 5 (1961), 198-205.

2. E. Artin, Elements of Algebraic Geometry, mimeographed notes, New York University, 1955.

3. R. Brauer, On the connection between the ordinary and modular characters of groups of finite order, Ann. of Math. 42 (1941), 926-935.

4. W. Burnside, Theory of Groups Finite Order, 2nd ed., Dover, New York, 1955.

5. C. W. Curtis and I. Reiner, Representation Theory of Finite Groups and Associative Algebras, Interscience, New York, 1962.

6. W. Feit, Groups which have a representation of degree less than $p$-1, Trans. Amer. Math. Soc. 112 (1964), 287-303.

7. M. Hall, Jr., Theory of Groups, Macmillan, New York, 1959.

8. I. M. Isaacs and D. S. Passman, Groups with representations of bounded degree, Canad. J. Math. 16 (1964), 299-309.

9 . Groups whose irreducible representations have degrees dividing $p^{e}$, Illinois J. Math. 8 (1964), 446-457.

10. N. Jacobson, Structure of Rings, Amer. Math. Soc. Colloquium Publications, vol. XXXVII.

11. A. G. Kurosh, The Theory of Groups, vol. II, 2nd English ed., Chelsea, New York 1960.

12. G. A. Miller, H. F. Blichfeldt and L. E. Dickson, Theory and Applications of Finite Groups, Dover, New York, 1961.

13. C. Rickart, Uniqueness of norm in Banach algebras, Ann. of Math. 51 (1950), 615-628.

14. A. Speiser, Die Theorie der Gruppen von Endlicher Ordnung, 4th ed., Birkhauser, Basel, 1956.

UNIVERSITY OF CHICAGO

University of CaLifornia, Los ANgeles 



\section{PACIFIC JOURNAL OF MATHEMATICS}

\section{EDITORS}

\author{
H. Samelson \\ Stanford University \\ Stanford, California \\ R. M. Blumenthal \\ University of Washington \\ Seattle, Washington 98105
}

\author{
J. Dugundu \\ University of Southern California \\ Los Angeles, California 90007 \\ *Richard Arens \\ University of California \\ Los Angeles, California 90024
}

\section{ASSOCIATE EDITORS}
E. F. BeCKENBACH
B. H. NeUMANN
F. WOLF
K. YosidA

\section{SUPPORTING INSTITUTIONS}

\author{
UNIVERSITY OF BRITISH COLUMBIA \\ CALIFORNIA INSTITUTE OF TECHNOLOGY \\ UNIVERSITY OF CALIFORNIA \\ MONTANA STATE UNIVERSITY \\ UNIVERSITY OF NEVADA \\ NEW MEXICO STATE UNIVERSITY \\ OREGON STATE UNIVERSITY \\ UNIVERSITY OF OREGON \\ OSAKA UNIVERSITY \\ UNIVERSITY OF SOUTHERN CALIFORNIA
}

\author{
STANFORD UNIVERSITY \\ UNIVERSITY OF TOKYO \\ UNIVERSITY OF UTAH \\ WASHINGTON STATE UNIVERSITY \\ UNIVERSITY OF WASHINGTON \\ * * * * \\ AMERICAN MATHEMATICAL SOCIETY \\ CALIFORNIA RESEARCH CORPORATION \\ SPACE TECHNOLOGY LABORATORIES \\ NAVAL ORDNANCE TEST STATION
}

Mathematical papers intended for publication in the Pacific Journal of Mathematics should by typewritten (double spaced). The first paragraph or two must be capable of being used separately as a synopsis of the entire paper. It should not contain references to the bibliography. No separate author's resumé is required. Manuscripts may be sent to any one of the four editors. All other communications to the editors should be addressed to the managing editor, Richard Arens, at the University of California, Los Angeles, California 90024.

50 reprints per author of each article are furnished free of charge; additional copies may be obtained at cost in multiples of 50 .

The Pacific Journal of Mathematics is published quarterly, in March, June, September, and December. Effective with Volume 13 the price per volume (4 numbers) is $\$ 18.00$; single issues, $\$ 5.00$. Special price for current issues to individual faculty members of supporting institutions and to individual members of the American Mathematical Society: $\$ 8.00$ per volume; single issues $\$ 2.50$. Back numbers are available.

Subscriptions, orders for back numbers, and changes of address should be sent to Pacific Journal of Mathematics, 103 Highland Boulevard, Berkeley 8, California.

Printed at Kokusai Bunken Insatsusha (International Academic Printing Co., Ltd.), No. 6, 2-chome, Fujimi-cho, Chiyoda-ku, Tokyo, Japan.

PUBLISHED BY PACIFIC JOURNAL OF MATHEMATICS, A NON-PROFIT CORPORATION

The Supporting Institutions listed above contribute to the cost of publication of this Journal, but they are not owners or publishers and have no responsibility for its content or policies.

* Basil Gordon, Acting Managing Editor until February 1, 1966. 


\section{Pacific Journal of Mathematics}

\section{Vol. 15, No. $3 \quad$ November, 1965}

David R. Arterburn and Robert James Whitley, Projections in the space of

bounded linear operators .................................

Robert McCallum Blumenthal, Joram Lindenstrauss and Robert Ralph Phelps,

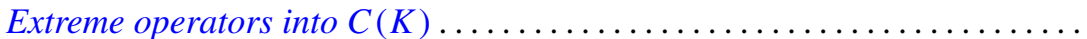

L. Carlitz, A note on multiple exponential sums ................... 757

Joseph A. Cima, A nonnormal Blaschke-quotient .................... 767

Paul Civin and Bertram Yood, Lie and Jordan structures in Banach algebras . . . 775

Luther Elic Claborn, Dedekind domains: Overrings and semi-prime

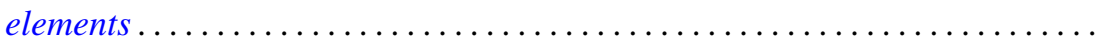

799

Luther Elic Claborn, Note generalizing a result of Samuel's .............. 805

George Bernard Dantzig, E. Eisenberg and Richard Warren Cottle, Symmetric

dual nonlinear programs ................................... 809

Philip J. Davis, Simple quadratures in the complex plane ............... 813

Edward Richard Fadell, On a coincidence theorem of F. B. Fuller ............ 825

Delbert Ray Fulkerson and Oliver Gross, Incidence matrices and interval

graphs ........................................ 835

Larry Charles Grove, Tensor products over $H^{*}$-algebras ..................

Deborah Tepper Haimo, $L^{2}$ expansions in terms of generalized heat polynomials

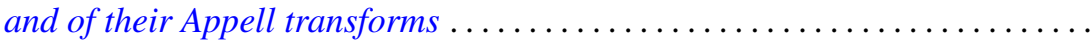

I. Martin (Irving) Isaacs and Donald Steven Passman, A chardcterization of groups in terms of the degrees of their characters ..........

Donald Gordon James, Integral invariants for vectors over local fields........ 905

Fred Krakowski, A remark on the lemma of Gauss ................... 917

Marvin David Marcus and H. Minc, A subdeterminant inequality ........... 921

Kevin Mor McCrimmon, Norms and noncommutative Jordan algebras ........ 925

Donald Earl Myers, Topologies for Laplace transform spaces ............... 957

Olav Njstad, On some classes of nearly open sets .................... 961

Milton Philip Olson, A characterization of conditional probability ........... 971

Barbara Osofsky, A counter-example to a lemma of Skornjakov .............. 985

Sidney Charles Port, Ratio limit theorems for Markov chains ............... 989

George A. Reid, A generalisation of $W^{*}$-algebras ...................... 1019

Robert Wells Ritchie, Classes of recursive functions based on Ackermann's

function ........................................... 1027

Thomas Lawrence Sherman, Properties of solutions of $n$th order linear

differential equations ........................................ 1045

Ernst Snapper, Inflation and deflation for all dimensions . .............. 1061

Kondagunta Sundaresan, On the strict and uniform convexity of certain Banach

spaces............................................. 1083

Frank J. Wagner, Maximal convex filters in a locally convex space .......... 1087

Joseph Albert Wolf, Translation-invariant function algebras on compact 